\title{
Time to peak tidal expiratory flow and the neuromuscular control of expiration
}

\author{
C.K. van der Ent*, C.P.M. van der Grinten+, N.E.L. Meessen+, S.C.M. Luijendijk+, \\ P.G.H. Mulder , J.M. Bogaard\#
}

Time to peak tidal expiratory flow and the neuromuscular control of expiration. C.K. van der Ent, C.P.M. van der Grinten, N.E.L. Meessen, S.C.M. Luijendijk, P.G.H. Mulder, J.M. Bogaard. CERS Journals Ltd 1998.

ABSTRACT: The ratio of the time needed to reach peak tidal expiratory flow ( $t$ PTEF) and the duration of expiration $(t \mathrm{E})$ is used to detect airflow obstruction in young children. $t$ PTEF is decreased in patients with asthma, but knowledge about the physiological determinants of this parameter is scarce. This study examined the relationship between $t$ PTEF and postinspiratory activities of inspiratory muscles and evaluated the effects of changing sensory information from the lung.

Airflow patterns and electromyographic (EMG) activity of inspiratory muscles were recorded in seven spontaneously breathing, anaesthetized cats. The trachea was cannulated and, as a result, the larynx and upper airways were bypassed. Changes in postinspiratory muscle activity were induced by changing afferent sensory nerve information (by cooling the vagus nerves, by administration of histamine and by additional application of continuous positive airway pressure (CPAP)).

Durations of postinspiratory activities of the diaphragm and intercostal muscles (characterized by their time constants $\tau_{\text {diaphr }}$ and $\tau_{\text {interc) }}$ correlated strongly with $t$ PTEF ( $\mathrm{r}=0.85$ and 0.77 , respectively). $\tau$ diaphr, $\tau$ interc and $t$ PTEF were significantly increased during cooling of the vagus nerves $\left(4-8^{\circ} \mathrm{C}\right)$ compared with values at 22 and $37^{\circ} \mathrm{C}(\mathbf{p}<0.05)$. Conversely, administration of histamine and CPAP caused significant decreases in $\tau_{\text {diaphr, }} \tau$ interc and $t$ PTEF, which were absent during cooling of the vagus nerves.

In conclusion, the time needed to reach peak tidal expiratory flow is highly influenced by the activities of inspiratory muscles during the early phase of expiration which, in turn, depend on the activities of vagal receptors in the lung.

Eur Respir J 1998; 12: 646-652.
*Wilhelmina Children's Hospital, University Hospital for Children and Youth, Dept of Pediatric Pulmonology, Utrecht, The Netherlands. +Dept of Pulmonology, University Hospital Maastricht, Maastricht University, Maastricht, The Netherlands. \$Dept of Epidemiology and Biostatistics, Erasmus University Rotterdam, Rotterdam, The Netherlands. "Pathophysiological Laboratory, Dept of Pulmonary Diseases, University Hospital Dijkzigt, Rotterdam, The Netherlands.

Correspondence: C.K. van der Ent

Dept of Pediatric Pulmonology

Wilhelmina Children's Hospital

University Hospital for Children and Youth

Department of Pediatric Pulmonology

P.O. Box 18009

3501 CA Utrecht

The Netherlands

Fax: 31302334825

Keywords: Cats

inspiratory muscles

tidal breathing analysis

Received: March 61997

Accepted after revision March 131998
Tidal breathing analysis is used as a tool to quantify airway obstruction in infants and children. The ratio of the time needed to reach peak tidal expiratory flow ( $t$ PTEF) and the duration of expiration ( $t \mathrm{E})$ are decreased in patients with asthma and cystic fibrosis [1-3]. $t$ PTEF is the most important determinant of changes in this ratio in children with asthma [4]. $t$ PTEF increases after the inhalation of a bronchodilator in asthmatics $[1,2]$ and decreases after bronchial challenge with methacholine $[1,5]$. Several authors have shown that the parameter $t \mathrm{E}$ is relatively stable in these patients $[6,7]$.

Until now, the relationship between the ratio $t \mathrm{PTEF} / t \mathrm{E}$ and airway diameter is unclear. It has been suggested that $t \mathrm{PTEF} / t \mathrm{E}$ reflects primarily neuromuscular control of expiration, which can be further influenced by changing pulmonary mechanics such as changing airflow resistance or lung compliance [8]. MoRRIs et al. [8] observed that postinspiratory activity of inspiratory muscles was decreased in patients with airflow obstruction. Activity of inspiratory muscles during the early phase of expiration causes braking of the expiratory airflow. A change in the postinspiratory activity of inspiratory muscles may, therefore, influence tPTEF. In an editorial, MiKkILINENI and ENGLAND [9] stressed the need for studies into the relationship between tidal breathing parameters and control of breathing.
This study was performed to elucidate the relationship between $t$ PTEF and the neuromuscular control of expiration. In an animal model investigations were made into: 1) the relationship between the parameter $t$ PTEF and postinspiratory activity of inspiratory muscles, and 2) the influence of afferent sensory vagus nerve information from the lung on $t$ PTEF. The results for $t$ PTEF were compared with those predicted by a model of the respiratory system.

\section{Methods}

\section{Study animals}

For this study experimental data were used that had been previously gathered by MEessen et al. [10] for a study into the effects of histamine and continuous positive airway pressure (CPAP) on end-tidal inspiratory muscle activity. The experimental procedures were described extensively in their study and will be summarized here.

The study was performed on seven adult cats (body weight $5.1 \pm 0.3 \mathrm{~kg}$ ), which were anaesthetized with ketamine-hydrochloride (10 mg.kg-1 i.m.) and a chloraloseurethane mixture $\left(12.5\right.$ and $62.5 \mathrm{mg} \cdot \mathrm{kg}^{-1} i . v$., respectively). 
To maintain surgical anaesthesia, supplemental doses of chloralose-urethane (5\% of initial dose) were given if needed. Body temperature was maintained between 36 and $38^{\circ} \mathrm{C}$. Both cervical vagus nerves were exposed in the mid-neck, freed from the carotid sheaths and cooled with the use of a Peltier element (range $37-4^{\circ} \mathrm{C} \pm 0.2^{\circ} \mathrm{C}$ ).

\section{Airflow recording}

The cats breathed spontaneously and were placed in the supine position on an operating table. The trachea was cannulated and connected to a pneumotachometer (Fleisch 0 Gould, Bilthoven, the Netherlands) to measure airflow. The other side of the Fleisch head was connected to a main tube in which a constant bias flow of $\sim 18 \mathrm{~L} \cdot \mathrm{min}^{-1}$ was maintained, to prevent rebreathing of expired air. With the help of an adjustable flow resistance in the bias flow a CPAP could be set.

From the flow recordings, $t \mathrm{E}, t \mathrm{PTEF}$ and the ratio $t \mathrm{PTEF} /$ $t$ E were determined.

\section{Electromyographic recording}

A pair of hooked needle electromyographic (EMG) electrodes was inserted into the costal part of the diaphragm and a second pair of electrodes into a parasternal intercostal muscle in the third or fourth intercostal space. The electrical activities of the diaphragm and intercostal muscles were amplified, filtered (150-3,000 Hz), rectified and fed into leaky integrators with a time constant of $50 \mathrm{~ms}$ (Neurolog, Digitimer, Welwyn Garden City, UK).

Signals representing integrated EMG activity of the diaphragm and intercostal muscles, airflow and temperature of the vagus nerves were monitored continuously and were sampled $(50 \mathrm{~Hz})$ with a computer (Compaq 386, Houston, TX, USA) and stored on the hard disk for offline analysis.

The measured EMG activities during the expiration were evaluated by fitting the integrated signals with the function $A e-t / \tau+B$, where $t$ is time, $A$ and $B$ are amplitudes and $\tau$ the time constant of the decay of inspiratory muscle activity ( $\tau$ diaphr for the diaphragm and $\tau$ interc for the intercostal muscles). A representative recording is presented in figure 1.

\section{Experimental protocol and background}

Changes in $\tau$ diaphr and $\tau$ interc were induced by changing afferent sensory nerve information from the lung. Vagal nerve receptors in the lung can be stimulated by the administration of histamine or CPAP. Intravenous histamine strongly stimulates rapidly adapting receptors (RAR) by a direct chemical effect [11-13], but also indirectly by mechanical stimulation when bronchoconstriction is induced. CPAP stimulates predominantly slowly adapting receptors (SAR) and, to a lesser extent RAR [14]. Conductance of vagal afferent activity can be inhibited and finally blocked by cooling both cervical vagus nerves. Accordingly, the following experimental protocol was used. After recording at least 10 baseline breathing cycles, $300 \mu \mathrm{g}$ histamine-diphosphate was administered intravenously. After the change in breathing pattern in response to histamine was apparent for about 20-30 s, CPAP of $0.9 \mathrm{kPa}$ was applied during 6-10 breathing cycles. A high level of CPAP was used to stimulate SAR forcefully. In this way,

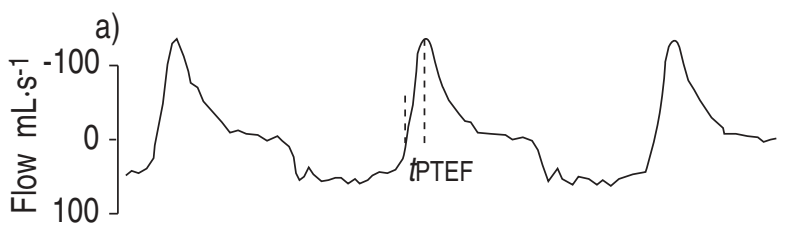

b)
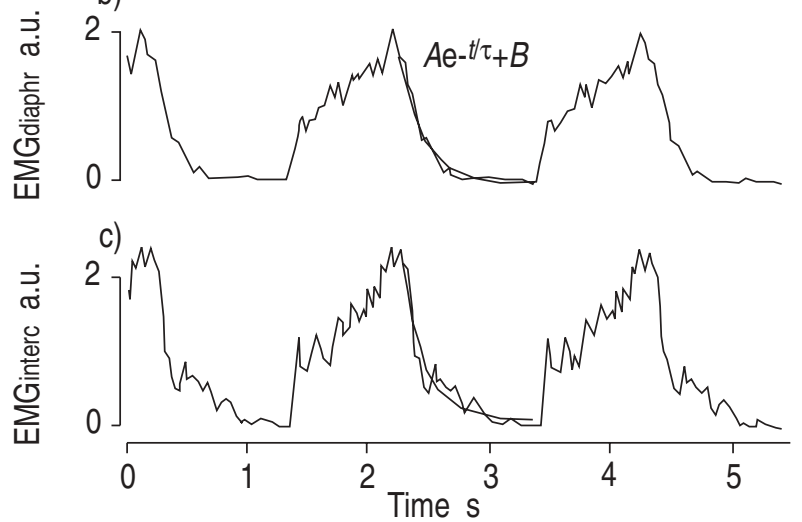

Fig. 1. - Representative recording of a) tidal breathing airflow with concomitant recordings of integrated electromyographic (EMG) activity of b) the diaphragm (diaphr) and c) the intercostal muscles (interc). Postinspiratory EMG activity was fitted with the function $A \mathrm{e}^{-t / \tau}+B$, as shown in the second breathing cycle. $t$ PTEF: time needed to reach peak tidal expiratory flow.

three runs of breathing cycles were recorded subsequently: 1) breathing cycles during control conditions; 2) breathing cycles after the administration of histamine, just before the application of CPAP; and 3) breathing cycles during histamine plus CPAP. All parameters were expressed as a mean value of six regular sequential breathing cycles. The protocol was carried out at the following temperatures of the vagus nerves: $37,22,14,12,10,8,6$ and $4^{\circ} \mathrm{C}$. Between two consecutive protocols a recovery period was allowed, until the breathing pattern had returned to the pattern prior to the administration of histamine. This protocol provided a wide range of $\tau$ values. After preparation of the cat, a typical experiment lasted for about $4 \mathrm{~h}$.

A simplified mechanical model of the respiratory system was adopted to compute $t$ PTEF as a function of $\tau$. This model consists of a single respiratory resistance $(R \mathrm{rs})$ and a single respiratory elastance $(E \mathrm{rs})$ in series (for details see Appendix).

\section{Statistical analysis}

All data are presented as mean \pm SEM. Because of the relatively small sample sizes and because data did not show normal distributions, a Wilcoxon test for paired observations was used to compare differences between baseline, histamine and histamine plus CPAP values and differences between values obtained at different temperatures. A p-value $<0.05$ was considered significant.

The relationships between $t$ PTEF and $\tau$ diaphr and between $t$ PTEF and $\tau$ interc were studied with a quadratic random coefficients model, based on the concave curvilinear appearance. In this model the dependent variable $t$ PTEF was related to the independent variables $\tau$ diaphr or $\tau$ interc as follows: 


$$
t \mathrm{PTEF}=b 0+b 1 \tau+b 2 \tau^{2}+\varepsilon .
$$

In this model the three coefficients $b 0, b 1$ and $b 2$ have a three-dimensional normal distribution across the cats with means $\beta 0, \beta 1$, and $\beta 2$ and a $3 \times 3$ covariance matrix. The variance of the residuals $\left(\sigma^{2} \varepsilon\right)$ was supposed to be equal in all cats. Subsequently, the multiple correlation coefficient $\mathrm{r}$ between $t$ PTEF and $\tau$ can be defined as follows:

$$
\mathrm{r}=1-\left(\sigma^{2} \varepsilon / \sigma^{2} \text { tot }\right)
$$

where $\sigma^{2}$ tot is the variance of all $t$ PTEF values.

\section{Results}

\section{Relationship between $\mathrm{tPTEF}$ and muscular activity}

Figure 2 shows a positive correlation between $t$ PTEF and $\tau$ diaphr. This relationship flattens off at higher values of $\tau$ diaphr. Therefore, a quadratic term $\left(b 2 \tau^{2}\right)$ was added to the linear equation $t \mathrm{PTEF}=b 0+b 1 \tau$, as described in the Methods section. This equation was fitted for each cat individually. The estimated mean coefficients $\beta_{0}, \beta_{1}$ and $\beta_{2}$ are shown in table 1 . The estimated mean regression curve is shown in figure 3 . The correlation between $t$ PTEF and $\tau$ diaphr was significant (multiple correlation coefficient $\mathrm{r}=0.85$ ).

The parameter $t$ PTEF also correlated significantly with $\tau_{\text {interc }}(r=0.77)$. The estimated mean coefficients $\beta 0, \beta 1$, and $\beta_{2}$ are shown in table 1 . The estimated mean regression curve for $t$ PTEF as a function of $\tau$ interc is shown in figure 3.

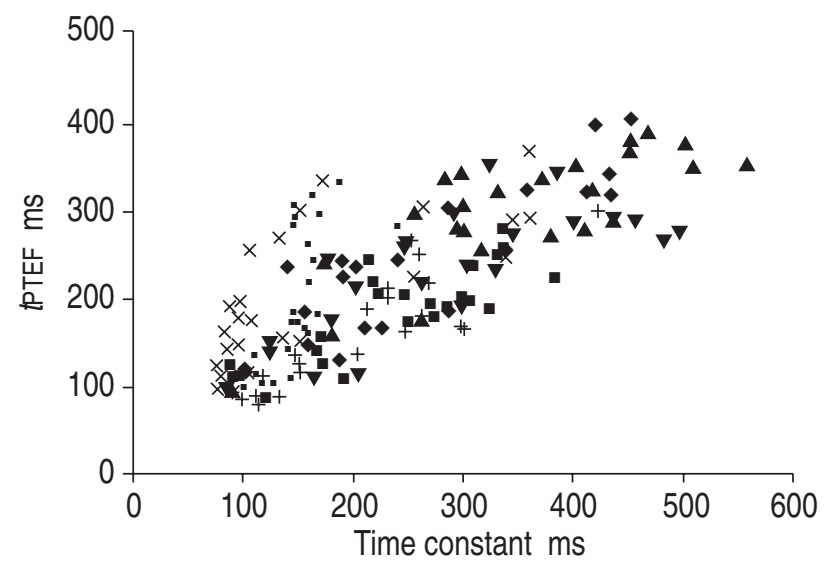

Fig. 2. - Relationship between decay of postinspiratory activity of the diaphragm (expressed as a time constant of the electromyographic signal decay, $\left.\tau_{\text {diaphr }}\right)$ and the time needed to reach peak tidal expiratory flow ( $t$ PTEF) in seven cats under different experimental conditions. The different symbols represent different animals.

Table 1. - Estimated coefficients of the equation tPTEF $=$ $b 0+b 1 \tau+b 2 \tau^{2}+\varepsilon$ for $\tau$ diaphr and $\tau$ interc in seven cats

\begin{tabular}{lcc}
\hline & $\tau$ diaphr & interc \\
\hline$\beta_{0} \mathrm{~ms}^{-1}$ & $25.7(28.9)$ & $40.3(22.3)$ \\
$\beta_{1}$ & $1.2214(0.3226)$ & $0.9788(0.1217)$ \\
$\beta_{2} \mathrm{~ms}^{-1}$ & $-1.2630 \times 10^{-3}\left(0.6518 \times 10^{-3}\right)$ & $-0.9006 \times 10^{-3}\left(0.1607 \times 10^{-3}\right)$ \\
\hline
\end{tabular}

Values are shown as mean \pm SEM. $t$ PTEF: time needed to reach peak tidal expiratory flow; $\tau$ diaphr: time constant of the decay of postinspiratory electromyographic (EMG) activity of the diaphragm; $\tau$ interc: time constant of the decay of postinspiratory EMG activity of the intercostal muscles. $\beta 0, \beta 1, \beta 2$ : means of the three coefficients $b 0, b 1$ and $b 2$.

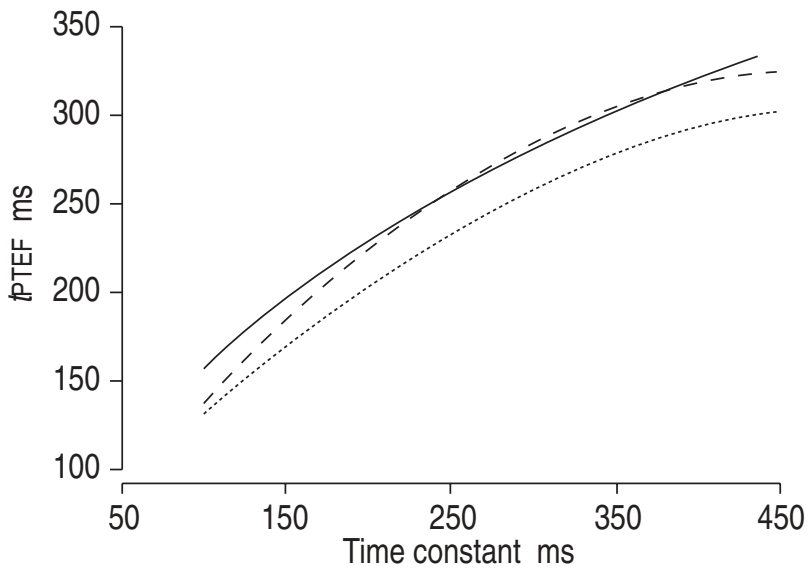

Fig. 3. - Estimated mean regression curves of the relationship between the time needed to reach peak tidal expiratory flow ( $t \mathrm{PTEF})$ and the time constant of the decay of postinspiratory electromyographic (EMG) activity of the diaphragm $\tau$ diaphr (- - - ) or the intercostal muscles $\tau$ interc (...... ) in seven anaesthetized cats._ : represents the relationship between $t$ PTEF and $\tau$ according to the mechanical model, with $\tau_{\mathrm{rs}}=$ 0.253 (see Equation A8 of the Appendix)

The model (see Appendix) was used to calculate $t$ PTEF as a function of $\tau$. For this calculation a time constant of the respiratory system $\left(\tau_{\mathrm{rs}}=R \mathrm{rs} / E \mathrm{rs}\right)$ of $0.253 \mathrm{~s}$ was used. This value has been reported as the mean value of six anaesthetized cats in a study of ZiN et al. [15]. The calculated $t$ PTEF as a function of $\tau$ is also shown in figure 3 .

\section{Influence of histamine}

At temperatures of the vagus nerves $>8^{\circ} \mathrm{C} \tau$ diaphr, $\tau_{\text {interc }}$ and $t$ PTEF decreased significantly after $i . v$. administration of histamine (table 2, figs. 4 and 5). At vagal temperatures of 4,6 and $8^{\circ} \mathrm{C}$ no significant decreases, or even small increases in these parameters were observed. The parameter $t$ E did not change significantly after the administration of histamine at vagal temperatures of 4 and $6^{\circ} \mathrm{C}$. At the higher temperatures, $t \mathrm{E}$ decreased significantly after histamine. The concordant changes in $t$ PTEF and $t$ E resulted in stable ratios for $t \mathrm{PTEF} / \mathrm{tE}$, without significant influence of the administration of histamine (table 2).

\section{Influence of histamine plus CPAP}

Application of CPAP caused a significant further decrease in $\tau$ diaphr compared with histamine without CPAP at vagal temperatures $<10^{\circ} \mathrm{C}$. At lower temperatures no significant changes were observed (fig. 4, table 2). CPAP caused a further decrease of $\tau$ interc at vagal temperatures of 22 and $37^{\circ} \mathrm{C}$. At temperatures of 4 and $6^{\circ} \mathrm{C}$ there was a nonsignificant decrease, while at temperatures of $8-14^{\circ} \mathrm{C}$ there was

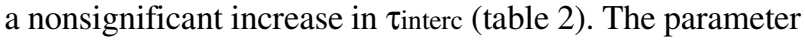
$t$ PTEF decreased at all vagal temperatures. This decrease was significant except at 12 and $14^{\circ} \mathrm{C}$ (fig. 5, table 2). $t \mathrm{E}$ was not influenced by the application of CPAP at 4 and $6^{\circ} \mathrm{C}$. At $8-14^{\circ} \mathrm{C}, t \mathrm{E}$ increased significantly compared with the histamine values and was comparable to the baseline values. At temperatures of 22 and $37^{\circ}$, $t$ E increased considerably to levels above the baseline values (table 2 ). 
Table 2. - Tidal breathing and inspiratory muscle electromyographic (EMG) parameters in seven cats under different experimental conditions (i.v. histamine and continuous positive airway pressure (CPAP) at different temperatures of the cervical vagus nerves)

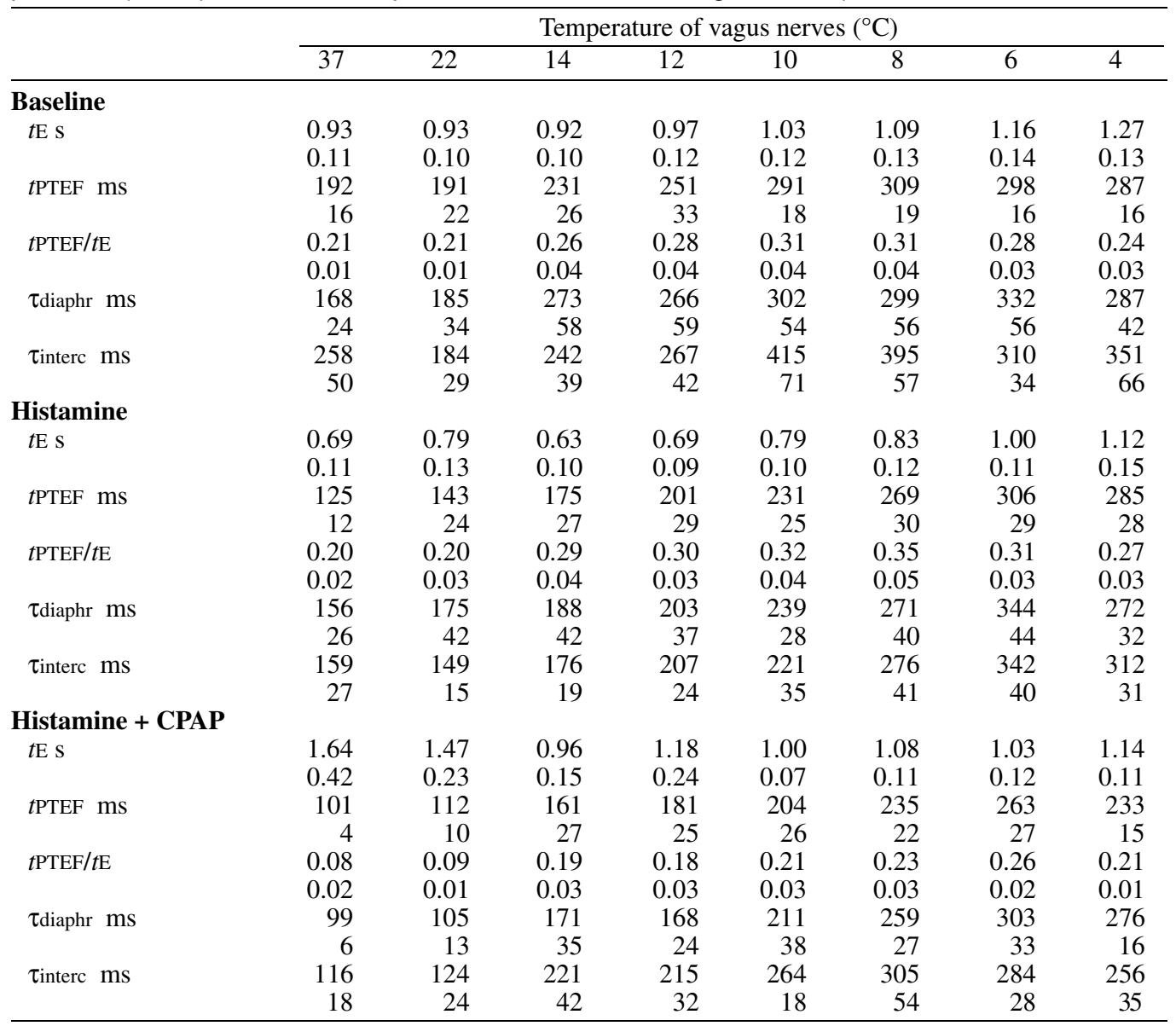

$t$ E: duration of expiration; $t$ PTEF: time needed to reach peak tidal expiratory flow; $\tau$ diaphr: time constant of the decay of postinspiratory EMG activity of the diaphragm; $\tau$ interc: time constant of the decay of postinspiratory EMG activity of the intercostal muscles.

Because of these changes the ratio $t \mathrm{PTEF} / \mathrm{tE}$ was significantly decreased during histamine plus CPAP at vagal temperatures above $8^{\circ} \mathrm{C}$. At lower temperatures no changes were observed (table 2).

\section{Influence of cooling of vagus nerves}

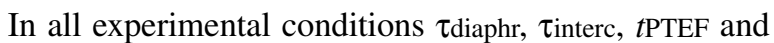
the ratio $t \mathrm{PTEF} / \mathrm{t}$ e were significantly lower at vagal temperatures of 22 and $37^{\circ} \mathrm{C}$ compared with their values at 4,6 and $8^{\circ} \mathrm{C}$ (table 2, figs. 4 and 5). No significant changes in baseline $t$ E values were observed at different vagal temperatures. The changes in $t \mathrm{E}$ induced by administration of histamine and CPAP were not observed at 4 and $6^{\circ} \mathrm{C}$.

\section{Discussion}

Relationship between $\mathrm{tPTEF}$ and inspiratory muscle activity

This study shows that $t$ PTEF correlated strongly with $\tau$ diaphr and $\tau$ interc. A rapid decay in the activities of the diaphragm and intercostal muscles during the first part of ex- piration correlates with low $t$ PTEF values (fig. 2). Changes in $\tau$ diaphr and $\tau_{\text {interc were induced by changing the afferent }}$ sensory nerve information from the lung. The considerable changes induced in $\tau_{\text {diaphr }}$ and $\tau_{\text {interc }}$ were followed closely followed by similar changes in $t$ PTEF (figs. 2 and 3 ). This suggests that the parameter $t$ PTEF depends strongly on the neuromuscular control of expiration.

The most important driving force of expiratory airflow is the elastic recoil of the respiratory system [16]. In paralysed subjects, after release of artificial inflation of the lungs, the expiratory airflow reaches a peak value almost immediately and is followed by an exponential decay [17, 18]. This decay can be described by $\tau_{\text {rs. }}$ Thus, in paralysed subjects $t$ PTEF is almost zero. In nonparalysed subjects, expiratory airflow is decreased by the counteracting activity of inspiratory muscles [8]. Therefore, inspiratory muscle activity during the first part of expiration can increase $t$ PTEF.

As shown in figure 1 , the decay of inspiratory muscle activity during expiration can be described by a monoexponential function $\left(A \mathrm{e}_{-}^{-t / \tau}+B\right)$. Therefore, the equation of motion of the respiratory system can be solved analytically (Appendix). A simple expression is obtained for $t$ PTEF as function of $\tau$ and $\tau$ rs (Equation A8 in the Appendix). This 


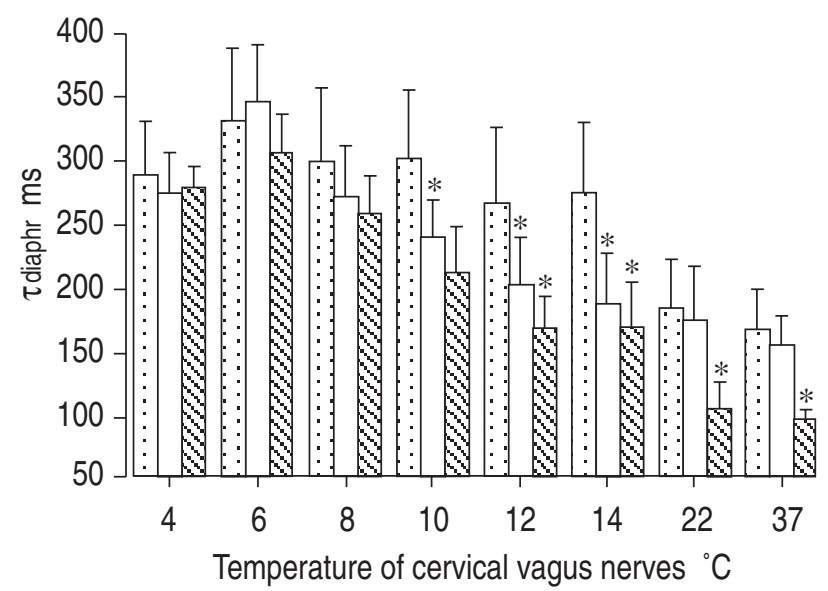

Fig. 4. - Velocity of decay of postinspiratory electromyographic (EMG) activity of the diaphragm (expressed as $\tau_{\text {diaphr }}$ in seven cats under different experimental conditions. The bars represent mean $\tau$ diaphr values ( $(\mathrm{SEM})$ at baseline ( 5 i:3), after i.v. histamine ( $\square$ ) and after histamine plus continuous positive airway pressure $(\measuredangle)$ at different temperatures of the cervical vagus nerves. $*: p<0.05$, significant difference from the previous condition at that temperature.

model shows that $\tau$ and $\tau_{\text {rs }}$ are equally important determinants of $t$ PTEF. The computed relationship between $t$ PTEF and $\tau$ (with $\tau_{\mathrm{rs}}=0.253 \mathrm{~s}$, the average value obtained in anaesthetized cats by ZiN et al. [15]) corresponds well with the experimentally observed relationships (fig. 3), with the best relationship for $\tau$ diaphr. This is in line with the fact that in the present experimental conditions the diaphragm is the most important inspiratory muscle.

In the model computations, a single value for $\tau_{\mathrm{rs}}(0.253$ s) was used for all cats and all experimental conditions. Studies in other mammalian species showed small changes in $\tau_{r s}$ after vagotomy and suggested a major influence of vagotomy on the neuromuscular control of breathing [19, $20]$. Despite the use of a single $\tau_{\text {rs }}$ value, a good correlation was observed between experimental data and the model results. In future studies, simultaneous measurements of $\tau_{\mathrm{rs}}$ may improve the validation of the model.

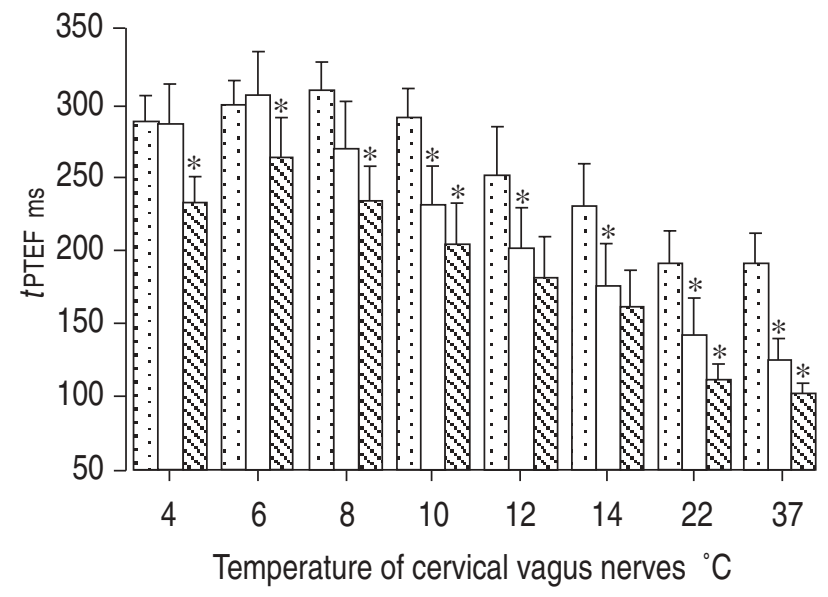

Fig. 5. - Time needed to reach peak tidal expiratory flow ( $t$ PTEF) in seven cats under different experimental conditions. The bars represent mean $t$ PTEF values ( $( \pm$ SEM) at baseline ( 1.9 , after i.v. histamine $(\square)$ and after histamine plus continuous positive airway pressure $(\square)$ at different temperatures of the cervical vagus nerves. $*: p<0.05$, significant difference from the previous condition at that temperature.
Many studies have shown that $t$ PTEF decreases in patients with airflow obstruction [1-5]. With regard to the present findings, this decrease in $t$ PTEF may be caused by a decrease in $\tau$ diaphr and $\tau_{\text {interc in these patients. Several }}$ studies have shown a more rapid decay of inspiratory muscle activity in patients with airway obstruction $[8,21]$.

In the present animal study, the main interest concerned the influence of postinspiratory activity of inspiratory muscles on $t$ PTEF. In human subjects expiratory muscles probably do not play an important role during quiet breathing. MoRRIs et al. [8] found EMG silence over expiratory abdominal muscles in adults with moderate to severe airflow obstruction. In children with asthma it may be supposed that intrinsic muscles of the larynx which control upper airway resistance will also influence expiratory airflow and $t$ PTEF. In the present study all animals were intubated to bypass the laryngeal mechanisms. According to the model, changes in upper airway resistance will result in changes in $\tau_{\text {rs }}$ and will, consequently, influence $t$ PTEF. Therefore, further studies into the role of the larynx and the interplay between the activities of laryngeal, inspiratory and expiratory muscles during early expiration in healthy and diseased subjects are needed.

\section{Influence of afferent vagus nerve information on $\mathrm{tPTEF}$}

This study showed that sensory information from the vagus nerves plays an important role in influencing $\tau$ diaphr

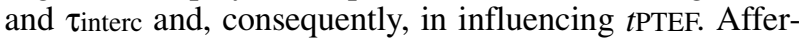
ent sensory vagus nerve information can be modulated by cooling the nerves or by stimulation of vagus nerves receptors. It has been shown that at vagal temperatures below $14^{\circ} \mathrm{C}$ conduction in myelinated fibres is progressively reduced and virtually absent at 4 and $6^{\circ} \mathrm{C}[22,23]$. These myelinated vagus nerve fibres transmit signals from rapidly and slowly adapting stretch receptors (RAR and SAR) in the lung [24].

In this study, changes in afferent vagus nerve activity were induced by administration of histamine and by additionally applied CPAP. Intravenous administration of histamine caused a significant decrease in $\tau_{\text {diaphr, }} \tau_{\text {interc }}$ and $t$ PTEF at vagal temperatures above $10^{\circ} \mathrm{C}$. At lower vagal temperatures no histamine-induced decrease in $t$ PTEF was observed (table 2). The application of CPAP after histamine induced a further decrease in $\tau$ diaphr, $\tau_{\text {interc }}$ and $t$ PTEF. Similarly, this decrease was not observed at the lowest temperatures of the vagus nerves (table 2). These data show that both the histamine and CPAP-induced changes depend on intact nerve conduction.

Cooling of the vagus nerves resulted in a significant increase of $\tau_{\text {diaphr }}$ and $\tau_{\text {interc }}$ (fig. 4 ). Baseline values of $\tau$ diaphr, Tinterc, and $t$ PTEF were significantly lower at temperatures of 22 and $37^{\circ} \mathrm{C}$ than at 4,6 and $8^{\circ} \mathrm{C}$. These data show that an increase in stimuli from the vagus nerves resulted in a decrease in the postinspiratory activity of inspiratory muscles.

Although one should be cautious in applying these findings in cats to children, vagal influence on expiratory braking mechanisms is also presumed to occur in the developing human [25]. Therefore, it might be speculated that differences in $\mathrm{PPTEF}$ between healthy children and asthmatic children with normal lung function [1] are caused by differences in afferent sensory nerve information from the lung. 
In conclusion, this study shows that the time needed to reach peak tidal expiratory flow is highly influenced by the activities of inspiratory muscles during the early phase of expiration which, in turn, depend partly on the activities of vagal receptors in the lung. In addition, the good agreement between experimental data and the model res-ults supports the view that the time needed to reach peak tidal expiratory flow is largely determined by the mechanical properties of the respiratory system in combination with the behaviour of inspiratory muscle activity during ex-piration.

\section{Appendix}

\section{Model and model equations}

Using a simplified mechanical model of the respiratory system the equation of motion can be written

$$
P(t)=R \operatorname{rs} V^{\prime}(t)+\operatorname{Ers} V(t)
$$

where $P(\mathrm{t})$ is the driving pressure at time $t, V(t)$ is the lung volume relative to relaxed lung volume, $V^{\prime}(t)$ represents the flow at time $t$, and $R \mathrm{rs}$ and $E$ rs are the resistance and elastance of the respiratory system, respectively [26]. In the case of passive expiration $P(t)=0$, where the solution of Equation (A1) yields an exponential decrease in $V$ with time with a time constant equal to that of the respiratory system $\left(\tau_{\mathrm{rs}}\right)$. In the absence of expiratory muscle activity $P(t)$ is solely the result of inspiratory muscle activity. For that case SiafaKas et al. [27] have shown in anaesthetized cats that $P(t)$ is nearly proportional to inspiratory muscle activity. This implies that if inspiratory muscle activity during expiration can be described by the function $A \mathrm{e}^{-t / \tau}+B$ (see Methods) the corresponding driving pressure will obey the relationship

$$
P(t)=\mathrm{P}_{1} \mathrm{e}^{-t / \tau}+\mathrm{P}_{2}
$$

where $P 1$ and $P 2$ are amplitudes and $t=0$ corresponds to the beginning of expiration. Substitution of Equation A2 into A1 results in

$$
P 1 \mathrm{e}^{-t / \tau}+P 2=R \operatorname{rs} V^{\prime}(t)+E \operatorname{rs} V(t)
$$

The general solution of this first-order differential equation can be written as:

$$
V(t)=E^{-1} \mathrm{rs}\left\{P 1\left(1-\tau_{\mathrm{rs}} / \tau\right)^{-1} \mathrm{e}^{-t / \tau}+P 2\right\}+C \mathrm{e}^{-t / \tau \mathrm{rs}}
$$

where $\tau_{\mathrm{rs}}=R \mathrm{rs} / E \mathrm{rs}$, and $C$ is a constant, the value of which is determined by the further boundary conditions. At the transition from inspiration to expiration the flow is zero, i.e. $V^{\prime}(t=0)=0$. According to Equations $\mathrm{A} 2-\mathrm{A} 4$ this results in

$$
\begin{gathered}
P(t=0)=P 1+P 2=E \mathrm{rs} V(t=0)=\left(1-\tau_{\mathrm{rs}} / \tau\right)^{-1} \\
P 1+P 2+C E \mathrm{rs}
\end{gathered}
$$

from which follows

$$
C=P 1\left\{1+\left(\tau_{\mathrm{rs}} / \tau-1\right)^{-1}\right\} E^{-1} \mathrm{rs}
$$

In this manuscript, the time that corresponds to peak tidal expiratory flow is denoted as $t$ PTEF. According to this model $t$ PTEF corresponds to the value of $t$ for which

$$
V^{\prime \prime}(t)=0
$$

where $V^{\prime \prime}(t)$ represents the second derivative of $V(t)$. After substitution of equation A6 into A4, $V^{\prime \prime}(t)$ can be calculated. Application of the condition $V^{\prime \prime}(\mathrm{t})=0$ for $t=t \mathrm{PTEF}$ results (after some mathematical manipulations) in the following relationship for $t \mathrm{PTEF}$ :

$$
t \mathrm{PTEF}=\left(1 / \tau-1 / \tau_{\mathrm{rs}}\right)-1 \ln \left(\tau_{\mathrm{rs}} / \tau\right)
$$

\section{References}

1. van der Ent CK, Brackel HJL, Van der Laag J, Bogaard JM. Tidal breathing analysis as a measure of airway obstruction in children aged three years and over. Am J Respir Crit Care Med 1996; 153: 1253-1258.

2. Carlsen KH, Lodrup-Carlsen KC. Tidal breathing analysis and response to salbutamol in awake young children with and without asthma. Eur Respir J 1992; 7: 21542159.

3. Stocks J, Dezateux CA, Jackson EA, Hoo A, Costeloe KL, Wade AM. Analysis of tidal breathing parameters in infancy: how variable is $t$ PTEF:tE? Am J Respir Crit Care Med 1994; 150: 1347-1354.

4. Lodrup Carlsen KC, Stenzler A, Carlsen KH. Do changes in $t \mathrm{PTEF} / t \mathrm{E}$ in health and disease in young children reflect different mechanisms? Eur Respir J 1995; 8: Suppl. 19, 57 s.

5. Benoist MR, Brouard JJ, Rufin P, Delacourt C, Waernessyckle S, Scheinmann P. Ability of new lung function tests to assess methacholine-induced airway obstruction in infants. Pediatr Pulmonol 1994; 18: 308-316.

6. Clarke JR, Aston H, Silverman M. Evaluation of a tidal expiratory flow index in healthy and diseased infants. Pediatr Pulmonol 1994; 17: 285-290.

7. Aston H, Clarke J, Silverman M. Are tidal breathing indices useful in infant bronchial challenge tests? Pediatr Pulmonol 1994; 17: 225-230.

8. Morris MJ, Madgwick RG, Frew AJ, Lane DJ. Breathing muscle activity during expiration in patients with chronic airflow obstruction. Eur Respir J 1990; 3: 901-909.

9. Mikkilineni S, England S. On tidal expiratory flow measurements in infants (Editorial). Pediatr Pulmonol 1994; 18: 71-72.

10. Meessen NEL, Van der Grinten CPM, Folgering HThM, Luijendijk SCM. Histamine-induced end-tidal inspiratory activity and lung receptors in cats. Eur Respir J 1995; 8: 2094-2103.

11. Matsumoto S. Effects of ammonia and histamine on lung irritant receptors in the rabbit. Respir Physiol 1989; 77: 301-308.

12. Vidru EH, Hahn HL, Nadel JA, Sampson SR. Mechanisms by which histamine stimulates rapidly adapting receptors in dog lungs. J Appl Physiol 1977; 43: 397-402.

13. Yu J, Roberts AM. Indirect effects of histamine on pulmonary rapidly adapting receptors in cats. Respir Physiol 1990; 79: 101-110. 
14. Coleridge HM, Coleridge JCG. Reflexes evoked from tracheobronchial tree and lungs. In: Cherniak NS, Widdicombe JG, eds. Handbook of Physiology. The Respiratory System: Control of Breathing. Vol. II. Bethesda, MD. American Physiological Society, 1986; pp. 395-429.

15. Zin WA, Pengelly LD, Milic-Emili J. Active impedance of respiratory system in anesthetized cats. $J$ Appl Physiol 1981; 53: 149-157.

16. Guyton AC. Textbook of Medical Physiology, 6th Edn. Philadelphia, PA, W.B. Saunders, 1981; pp. 516-528.

17. Kavan EM, Haddy FJ. A study of the mechanics of respiration in the human being: preliminary report. Curr Res Anaesth Analg 1956; 35: 343-349.

18. Brody AW. Mechanical compliance and resistance of the lung-thorax calculated from the flow during passive expiration. Am J Physiol 1954; 178: 189-196.

19. Colebatch HJH, Halmagyi DFJ. Effect of vagotomy and vagal stimulation on lung mechanics and circulation. $J$ Appl Physiol 1963; 18: 881-887.

20. Caldeira MPR, Saldiva PHN, Zin WA. Vagal influences on respiratory mechanics, pressures, and control in cats. Respir Physiol 1988; 73: 43-53.

21. Citterio GE, Agostoni E, DelSanto A, Marazzini L.
Decay of inspiratory muscle activity in chronic airways obstruction. J Appl Physiol 1981; 51: 1388-1397.

22. Pisarri TE, Yu J, Coleridge HM, Coleridge JCG. Background activity in pulmonary vagal C-fibers and its effects on breathing. Respir Physiol 1986; 64: 29-43.

23. Jonzon A, Pisarri TE, Roberts AM, Coleridge JCG, Coleridge HM. Attenuation of pulmonary afferent input by vagal cooling in dogs. Respir Physiol 1988; 72: 19-34.

24. Sant'Ambrogio G. Information arising from the tracheobronchial tree of mammals. Physiol Rev 1982; 62: 531569.

25. Kosch PC, Hutchison AA, Wozniak JA, Carlo WA, Star AR. Posterior cricarytenoid and diaphragm activities during tidal breathing in neonates. J Appl Physiol 1988; 64: 1968-1978.

26. Agostoni E. Dynamics. In: Campbell EJM, Agostoni E, Newsom Davis J, eds. The Respiratory Muscles. Mechanisms and Neural Control, 2nd ed. London, Lloyd-Luke, 1970; 80-114.

27. Siafakas NM, Peslin R, Bonora M, Gautier H, Duron B, Milic-Emili J. Phrenic activity, respiratory pressures, and volume changes in cats. J Appl Physiol 1981; 51: 109121. 